\title{
天然番荔枝内酯类化合物构效关系研究进展
}

\author{
陈勇李 祥* 陈建伟 \\ (南京中医药大学药学院 南京 210046)
}

摘要 番荔枝内酯类化合物化学结构及抗肿瘤作用机制独特. 综述了这类天然化合物的构效关系研究进展.

关键词 番荔枝内酯; 构效关系; 进展

\section{Progress in Structure Activity Relationship of Natural Annonaceous Acetogenins}

\author{
Chen, Yong Li, Xiang* Chen, Jianwei \\ (College of Pharmaceutical Science, Nanjing University of Chinese Medicine, Nanjing 210046)
}

\begin{abstract}
Annonaceous acetogenins are characterized by special chemical structures and antitumor mechanism. In this paper, the advance in structure activity relationship of natural annonaceous acetogenins is reviewed.

Keywords annonaceous acetogenin; structure activity relationship; progress
\end{abstract}

1982 年 Jolad 等 ${ }^{[1]}$ 从番荔枝科紫玉盘属植物 Uvaria acuminata 根中分离得到第 1 个番荔枝内酯类化合物 (Annonaceous acetogenins, ACGs) Uvaricin, 它对小鼠白 血病细胞有强杀伤作用, 由此掀起了在番荔枝科植物中 分离这类化合物的高潮. 天然番荔枝内酯大都有不同程 度的抗肿瘤活性, 其中报道活性最强的 4 个化合物 bullatacin, asimicin, trilobacin 和 trilobin 对某些瘤株的 $\mathrm{IC}_{50}$ 达到或小于 $10^{-12} \mu \mathrm{g} / \mathrm{mL}^{[2]}$. 番荔枝内酯类化合物结 构中具有 $0 \sim 3$ 个四氢呋喃环(THF), 末端具有甲基取代 或经重排的 $\gamma$-内酯末端和具有羟基取代或末取代的长 直链烷基为特征, 碳原子数目多为 33 37 个, 番荔枝内 酯类化合物主要结构类型的结构分类见图 1 .

在过去的 20 多年, 国内外学者从番荔枝科植物中 共分离得到了近 500 个番荔枝内酯，同时很多番荔枝内 酯和番荔枝内酯衍生物也成功的合成 ${ }^{[3,4]}$, 番荔枝内酯 的抗癌活性的作用机理是通过抑制线粒体复合酶 I 使癌 细胞 ATP 的生成减少导致肿瘤细胞的凋亡 ${ }^{[5]}$. 结构中的 末端内酯环被认为是直接作用于线粒体复合酶 I. 在学 者 Miyoshi, Oberlies, He, Alali, Landolt, Gu, Alfonso 和
Delgi Esposti 等 ${ }^{[6 \sim 13]}$ 的研究基础上，大体概括了番荔枝 内酯的构效关系规律：(1)邻双四氢呋喃型 (adjacent bis-THF)番荔枝内酯的抗肿瘤活性强于间双四氢呋喃型 (non adjacent bis-THF) 番荔枝内酯和单四氢呋喃型 (mono-THF) 番荔枝内酯; (2)大部分间双四氢呋喃番荔 枝内酯对某些肿瘤细胞的抑制活性比单四氢呋喃番荔 枝内酯强，但某些单四氢呋喃型番荔枝内酯比间双四氢 呋喃型番荔枝内酯对某些肿瘤细胞具有更强的选择活 性.

但番荔枝内酯具有很多手性中心，碳链长短不一， 羟基和四氢呋喃环的个数位置及构型的不同，且其具有 较大的毒性. 因此, 番荔枝内酯的药理活性和结构的关 系，毒性和立体结构的关系及活性与毒性之间的关系值 得进一步研究. 本文从以下几个影响因素来综述天然的 番荔枝内酯化合物构效关系: (1)碳链长短的影响: 包括 连接两个环之间碳链及未端碳链的长度; (2)羟基的影 响: 包括羟基个数及取代位置及其构型; (3)四氢呋喃环 的影响: 包括四氢呋喃环个数及取代位置及其构型. 本 综述涉及到的化合物见表 1 .

\footnotetext{
*E-mail: lixiang_8182@163.com

Received September 4, 2011; revised November 17, 2011; published online January 7, 2012.

Project supported by the Priority Academic Program Development of Jiangsu Higher Education Institutions (No. ysxk-2010), the Doctoral Fund of Ministry of Education of China (No. 20113237110009), the Jiangsu Key Laboratory for Pharmacology and Safety Evaluation of Chinese Materia Medica (No. P09018) and the 2011 Program Sponsored for Scientific Innovation Research of College Graduate in Jiangsu Province (No. 790). 江苏省高校优势学科建设工程(No. ysxk-2010)、国家教育部博士点专项基金(No. 20113237110009)、江苏省中药药效与安全性评价重点实验室(No. P09018)开放研究课题和 2011 江苏省普通高校研究生科研创新计划(No. 790)资助项目.
} 


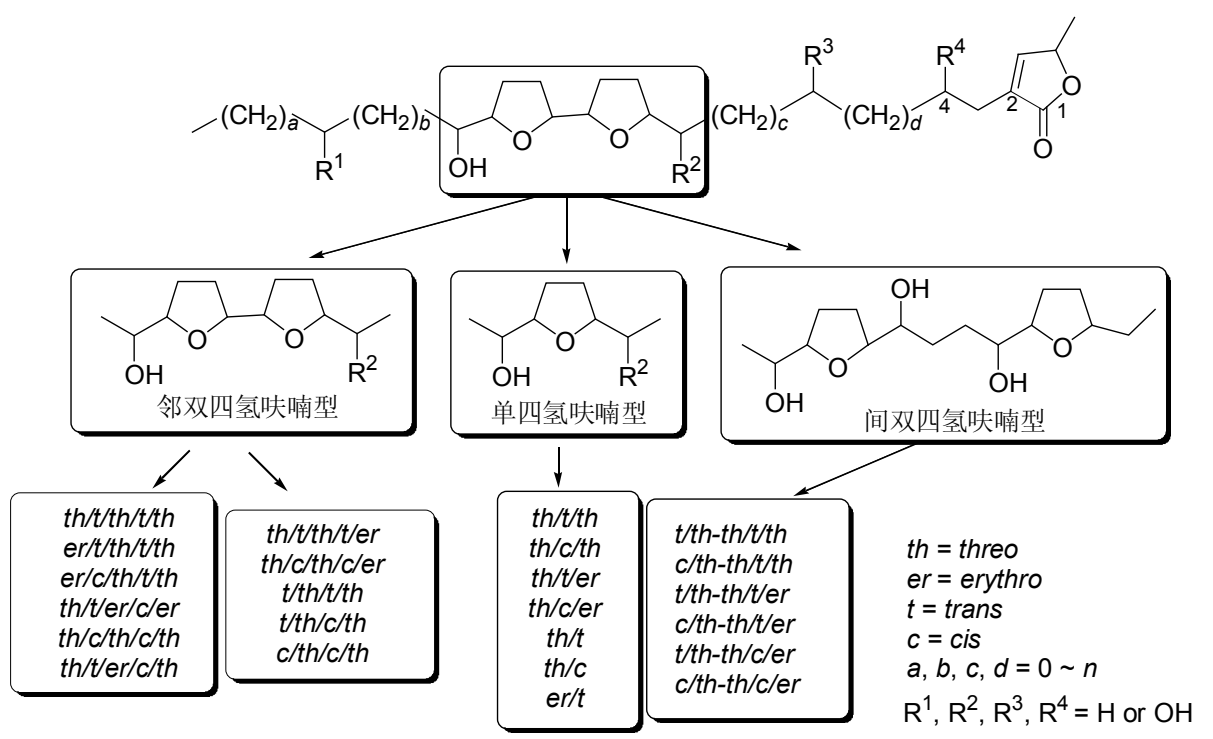

图 1 番荔枝内酯类化合物主要结构类型的结构分类

Figure 1 Structure of annonaceous acetogenins

表 1 本综述所涉及到的化合物

Table 1 Compounds in this paper

\begin{tabular}{|c|c|c|c|c|c|c|c|}
\hline 化合物编号 & 化合物名称 & 羟基位置 & THF 环构型 & 分子式 & 分子量 & 种属 ${ }^{a}$ & 参考文献 \\
\hline 1 & Montalicin-A & $4,13,18$ & th/t/th & $\mathrm{C}_{33} \mathrm{H}_{60} \mathrm{O}_{6}$ & 552 & A. montana & [14] \\
\hline 2 & Montalicin-B & $4,13,18$ & th/t/th & $\mathrm{C}_{35} \mathrm{H}_{64} \mathrm{O}_{6}$ & 580 & A. montana & [14] \\
\hline 3 & Montalicin-C & $4,7,13,18$ & th/t/th & $\mathrm{C}_{35} \mathrm{H}_{64} \mathrm{O}_{7}$ & 596 & A. montana & [14] \\
\hline 4 & Montalicin-E & $4,7,13,18$ & $t h / t / t h$ & $\mathrm{C}_{37} \mathrm{H}_{66} \mathrm{O}_{7}$ & 622 & A. montana & [14] \\
\hline 5 & Asitrilobin-A & $4,10,17,22$ & th/cler & $\mathrm{C}_{37} \mathrm{H}_{68} \mathrm{O}_{7}$ & 624 & As. triloba & {$[15]$} \\
\hline 6 & Asitrilobin-B & $4,10,15,20$ & th/cler & $\mathrm{C}_{35} \mathrm{H}_{64} \mathrm{O}_{7}$ & 596 & As. triloba & [15] \\
\hline 7 & Muricin-A & $4,19,26,27$ & t/th-th & $\mathrm{C}_{35} \mathrm{H}_{64} \mathrm{O}_{7}$ & 596 & A. muricata & [16] \\
\hline 8 & Muricin-C & $4,21,24,25$ & t/th-th & $\mathrm{C}_{35} \mathrm{H}_{64} \mathrm{O}_{7}$ & 596 & A. muricata & [16] \\
\hline 9 & Muricin-D & $4,19,22,23$ & t/th-th & $\mathrm{C}_{33} \mathrm{H}_{60} \mathrm{O}_{7}$ & 568 & A. muricata & [16] \\
\hline 10 & Carolin-A & $15,24,28$ & th/t/th/c/er & $\mathrm{C}_{37} \mathrm{H}_{66} \mathrm{O}_{7}$ & 622 & A. spinescens & [17] \\
\hline 11 & Carolin-C & $13,22,26$ & th/t/th/c/er & $\mathrm{C}_{35} \mathrm{H}_{62} \mathrm{O}_{7}$ & 594 & A. spinescens & [17] \\
\hline 12 & Goniothalamicin & $4,10,13,18$ & $t h / t / t h$ & $\mathrm{C}_{35} \mathrm{H}_{64} \mathrm{O}_{7}$ & 596 & A. montana & [14] \\
\hline 13 & Montalicin-D & $4,11,13,18$ & $t h / t / t h$ & $\mathrm{C}_{35} \mathrm{H}_{64} \mathrm{O}_{7}$ & 596 & A. montana & [14] \\
\hline 14 & Annonacin & $4,10,15,20$ & $t h / t / t h$ & $\mathrm{C}_{35} \mathrm{H}_{64} \mathrm{O}_{7}$ & 596 & A. montana & [14] \\
\hline 15 & Xylopianin & $4,8,15,20$ & $t h / t / t h$ & $\mathrm{C}_{35} \mathrm{H}_{64} \mathrm{O}_{7}$ & 596 & A. montana & [14] \\
\hline 16 & Annomuricin-E & $4,10,11,15,20$ & er-th/t/th & $\mathrm{C}_{35} \mathrm{H}_{64} \mathrm{O}_{8}$ & 612 & A. muricata & [18] \\
\hline 17 & Muricapentocin & $4,8,12,15,20$ & $t h / t / t h$ & $\mathrm{C}_{35} \mathrm{H}_{64} \mathrm{O}_{8}$ & 612 & A. muricata & [18] \\
\hline 18 & Muricin-B & $4,19,26,27$ & t/th-th & $\mathrm{C}_{35} \mathrm{H}_{64} \mathrm{O}_{7}$ & 596 & A. muricata & [16] \\
\hline 19 & Corossolin & $10,15,20$ & $t h / t / t h$ & $\mathrm{C}_{35} \mathrm{H}_{64} \mathrm{O}_{6}$ & 580 & A. muricata & [16] \\
\hline 20 & Corossolone & 15,20 & $t h / t / t h$ & $\mathrm{C}_{35} \mathrm{H}_{62} \mathrm{O}_{6}$ & 578 & A. muricata & [16] \\
\hline 21 & Rollitacin & $15,24,28,29$ & th/t/th/t/er-er & $\mathrm{C}_{37} \mathrm{H}_{66} \mathrm{O}_{8}$ & 638 & R. mucosa & [19] \\
\hline 22 & Purpurenin & $10,15,24,29$ & th/t/th/t/er & $\mathrm{C}_{37} \mathrm{H}_{66} \mathrm{O}_{8}$ & 638 & R. mucosa & [19] \\
\hline 23 & Annoglaucin & $4,10,15,24$ & th/t/th/t/er & $\mathrm{C}_{37} \mathrm{H}_{66} \mathrm{O}_{8}$ & 638 & R. mucosa & [19] \\
\hline 24 & Carolin-B & $15,24,29$ & th/t/th/cler & $\mathrm{C}_{37} \mathrm{H}_{66} \mathrm{O}_{7}$ & 622 & A. spinescens & [17] \\
\hline 25 & Spinencin & $15,24,28,29$ & th/t/th/cler-th & $\mathrm{C}_{37} \mathrm{H}_{66} \mathrm{O}_{8}$ & 638 & A. spinescens & [17] \\
\hline 26 & Squamocin & $15,24,28$ & th/t/th/t/er & $\mathrm{C}_{37} \mathrm{H}_{66} \mathrm{O}_{7}$ & 622 & A. squamosa & [20] \\
\hline 27 & Squamocin- $\mathrm{O}_{1}$ & $12,15,24,28$ & th/t/th/t/er & $\mathrm{C}_{37} \mathrm{H}_{66} \mathrm{O}_{8}$ & 638 & A. squamosa & [20] \\
\hline 28 & Squamocin- $\mathrm{O}_{2}$ & $12,15,24,28$ & th/t/th/t/er & $\mathrm{C}_{37} \mathrm{H}_{66} \mathrm{O}_{8}$ & 638 & A. squamosa & [20] \\
\hline 29 & Glabracin-A & $4,10,23,24$ & th/t/th/t-er & $\mathrm{C}_{37} \mathrm{H}_{66} \mathrm{O}_{8}$ & 638 & A. glabra & [21] \\
\hline 30 & Glabracin-B & $4,10,23,24$ & $t h / t / t h / t-t h$ & $\mathrm{C}_{37} \mathrm{H}_{66} \mathrm{O}_{8}$ & 638 & A. glabra & [21] \\
\hline 31 & Desacetyluvaricin & 15,24 & th/t/th/t/er & $\mathrm{C}_{37} \mathrm{H}_{66} \mathrm{O}_{6}$ & 606 & A. muricata & {$[22,23]$} \\
\hline
\end{tabular}




\begin{tabular}{|c|c|c|c|c|c|c|c|}
\hline 化合物编号 & 化合物名称 & 羟基位置 & THF 环构型 & 分子式 & 分子量 & 种属 $^{a}$ & 参考文献 \\
\hline 32 & Bullatacin & $4,15,24$ & th/t/th/t/er & $\mathrm{C}_{37} \mathrm{H}_{66} \mathrm{O}_{7}$ & 622 & R. mисоsa & {$[22,23]$} \\
\hline 33 & Annoglaucin & $4,10,15,24$ & th/t/th/t/er & $\mathrm{C}_{37} \mathrm{H}_{66} \mathrm{O}_{8}$ & 638 & A. muricata & [22] \\
\hline 34 & Rollimusin & $10,15,24,28$ & th/t/th/t/er & $\mathrm{C}_{37} \mathrm{H}_{66} \mathrm{O}_{8}$ & 638 & A. muricata & [22] \\
\hline 35 & Annonacin-A & $4,10,15,20$ & th/t/er & $\mathrm{C}_{35} \mathrm{H}_{64} \mathrm{O}_{7}$ & 596 & R. mucosa & [19] \\
\hline 36 & Longifolicin & $10,13,18$ & $t h / t / t h$ & $\mathrm{C}_{35} \mathrm{H}_{64} \mathrm{O}_{6}$ & 580 & A. muricata & {$[16]$} \\
\hline 37 & Muricatetrocins- $A+B$ & $4,16,19,20$ & t/th-th & $\mathrm{C}_{35} \mathrm{H}_{64} \mathrm{O}_{7}$ & 596 & A. muricata & [16] \\
\hline 38 & 4-Deoxyannoreticuin & $9,15,20$ & $t h / t / t h$ & $\mathrm{C}_{35} \mathrm{H}_{64} \mathrm{O}_{6}$ & 580 & A. squamosa & {$[24]$} \\
\hline 39 & cis-4-Deoxyannoreticuin & $9,15,20$ & $t h / c / t h$ & $\mathrm{C}_{35} \mathrm{H}_{64} \mathrm{O}_{6}$ & 580 & A. squamosa & [24] \\
\hline 40 & Calamistrin-C & $13,19,24$ & $t h / t / t h$ & $\mathrm{C}_{37} \mathrm{H}_{68} \mathrm{O}_{6}$ & 608 & U. calamistrata & {$[25]$} \\
\hline 41 & Calamistrin-D & $13,19,24$ & th/t/er & $\mathrm{C}_{37} \mathrm{H}_{68} \mathrm{O}_{6}$ & 608 & U. calamistrata & [25] \\
\hline 42 & Annoreticuin & $4,9,15,20$ & $t h / t / t h$ & $\mathrm{C}_{35} \mathrm{H}_{64} \mathrm{O}_{7}$ & 596 & A. montana & {$[14]$} \\
\hline 43 & cis-Annoreticuin & $4,9,15,20$ & $t h / c / t h$ & $\mathrm{C}_{35} \mathrm{H}_{64} \mathrm{O}_{7}$ & 596 & A. montana & [14] \\
\hline 44 & Annoglacin-A & $4,12,17,22$ & th/t/er & $\mathrm{C}_{37} \mathrm{H}_{68} \mathrm{O}_{7}$ & 624 & A. glabra & {$[26]$} \\
\hline 45 & Annoglacin-B & $4,12,17,22$ & $t h / t / t h$ & $\mathrm{C}_{37} \mathrm{H}_{68} \mathrm{O}_{7}$ & 624 & A. glabra & {$[26]$} \\
\hline 46 & Montacin & $4,9,20,25$ & $t h / t / t h$ & $\mathrm{C}_{35} \mathrm{H}_{62} \mathrm{O}_{8}$ & 610 & A. montana & {$[27]$} \\
\hline 47 & cis-Montacin & $4,9,20,25$ & $t h / c / t h$ & $\mathrm{C}_{35} \mathrm{H}_{62} \mathrm{O}_{8}$ & 610 & A. montana & {$[27]$} \\
\hline 48 & Purpuracenin & $4,10,15,24$ & th/clth/cler & $\mathrm{C}_{37} \mathrm{H}_{66} \mathrm{O}_{8}$ & 638 & A. purpurea & {$[28]$} \\
\hline 49 & Calamistrin-F & $5,17,26$ & $t h / t / t h / t / t h$ & $\mathrm{C}_{37} \mathrm{H}_{66} \mathrm{O}_{7}$ & 622 & U. calamistrata & {$[25]$} \\
\hline 50 & Calamistrin-G & $5,17,26$ & th/t/th/t/er & $\mathrm{C}_{37} \mathrm{H}_{66} \mathrm{O}_{7}$ & 622 & U. calamistrata & {$[25]$} \\
\hline 51 & Annocatacin-A & 4,23 & t/th/t/th & $\mathrm{C}_{35} \mathrm{H}_{62} \mathrm{O}_{6}$ & 578 & A. muricata & {$[22]$} \\
\hline 52 & Annocatacin-B & 4,23 & $c / t h / c / t h$ & $\mathrm{C}_{35} \mathrm{H}_{62} \mathrm{O}_{6}$ & 578 & A. muricata & {$[22]$} \\
\hline 53 & Asimicin & $4,15,24$ & $t h / t / t h / t / t h$ & $\mathrm{C}_{37} \mathrm{H}_{66} \mathrm{O}_{7}$ & 622 & A. muricata & {$[22]$} \\
\hline 54 & 12,15-cis-Squamostatin-D & $16,19,24$ & $c / t h$-th/t/er & $\mathrm{C}_{37} \mathrm{H}_{66} \mathrm{O}_{7}$ & 622 & A. atemoya & {$[23]$} \\
\hline 55 & Squamostatin-D & $16,19,24$ & t/th-th/t/er & $\mathrm{C}_{37} \mathrm{H}_{66} \mathrm{O}_{7}$ & 622 & A. atemoya & {$[23]$} \\
\hline
\end{tabular}

我们从报道的文献中总结构效关系时, 在考察上述 某一因素对构效关系的影响时都只选择其它两个因素 一致的化合物进行总结.

\section{1 番荔枝内酯类化合物的构型}

\section{1 末端内酯环的构型}

在目前分离得到的天然番荔枝内酯化合物其末端 内酯环有 7 种基本类型 ${ }^{[2]}$, 最常见的一种如图 1 所示, 其 4 位绝对构型均为 $S$.

\section{2 四氢呋喃环的构型}

\section{2 .1 单四氢呋喃型}

分为 $\alpha, \alpha^{\prime}$-二羟基取代 $\left(\alpha, \alpha^{\prime}\right.$-dihydroxylated)番荔枝内 酯和 $\alpha$ 或者 $\alpha^{\prime}$-单羟基取代(monodroxylated)番荔枝内 酯[2]. 其相对构型见图 1、绝对构型见表 2 .

\subsection{2 邻位双四氢呋喃型}

THF 两侧各有一个羟基相邻的相对构型有 th/t/th/t/th, er/t/th/t/th, er/c/th/t/th, th/tter/cler, th/c/th/c/th, th/t/er/c/th, $t h / t / t h / t / e r, t h / c / t h / c / e r$ 八种; 四氢呋喃环只有

一侧有羟基相邻的相对构型有 $t / t h / t / t h, t / t h / c / t h, c / t h / c / t h$ 三种 ${ }^{[2]}$ (图 1).

\subsection{3 间位双四氢呋喃型}

其相对构型有 $t / t h-t h / t / t h, c / t h$-th/t/th, t/th-th/t/er，
表 $2 \alpha, \alpha^{\prime}$-二羟基取代番荔枝内酯和 $\alpha$ 或者 $\alpha^{\prime}$-单羟基取代番荔 枝内酯及其构型

Table 2 Configurations of $\alpha, \alpha^{\prime}$-dihydroxylated ACGs and $\alpha$ or $\alpha$-hydroxylated ACGs

\begin{tabular}{ccc}
\hline Mono-THF 类 & 相对构型 $^{a}$ & 绝对构型 \\
\hline \multirow{4}{*}{$\alpha, \alpha^{\prime}$-Dihydroxylated } & th/t/th & $R, R$ \\
& th/c/th & $R, S$ \\
& th/t/er & $R, R$ \\
& $t h / c / e r$ & $R, S$ \\
& $t h / t$ & $R, S$ \\
$\alpha$ or $\alpha^{\prime}$-Dihydroxylated & $t h / c$ & $S, S$ \\
& $e r / t$ & $R, S$ \\
\hline
\end{tabular}

$a_{\text {th }}=$ threo $;$ er $=$ erythro $;=$ trans $; c=$ cis.

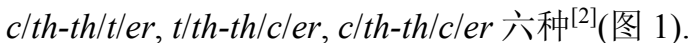

\section{2 构效关系}

\section{1 碳链长短的影响}

Liaw 等 ${ }^{[14]}$ 从 Annona montana 中分离出 $\alpha, \alpha^{\prime}$-二羟基 取代单四氢呋喃类型化合物 montalicin-A (1), montalicin-B (2), montalicin-C (3) 和 montalicin-E (4), 并测试 了它们对人肝癌细胞 Hep G2 及 Hep 3B 的活性. 其中 $\mathbf{1}$ 和 2 都为 4, 13, 18-OH 取代且四氢呋喃环的构型都为 th/t th, 唯一的不同之处在于 $\mathbf{1}$ 的末端碳链上碳数比 $\mathbf{2}$ 少 两个; 3 和 4 都为 4,7,13,18-OH 取代且四氢呋喃环的构型 
都为 $t h / t / t h$, 唯一的不同之处也在于前者比后者的末端 碳链上碳数少 2 个, 在比较这四个化合物的药效数据, 发现末端碳数少的化合物 $\mathbf{1}$ 和 $\mathbf{3}$ 的活性都比相应的 $\mathbf{2}$ 和 4 要强.

Woo 等 ${ }^{[15]}$ 从 Asimina triloba 中分离得到两个 $\alpha, \alpha^{\prime}$ 二羟基取代单四氢呋喃类型番荔枝内酯类化合物 asitrilobin-A (5)和 asitrilobin-B (6), 它们结构的不同之 处在于化合物 $\mathbf{5}$ 在两个环之间有 15 个碳原子, 而化合物 6 只有 13 个, 在对六种人肿瘤细胞的活性测试中发现化 合物 6 对这六种细胞的活性均强于化合物 5. 而从文献 报道 ${ }^{[14,16]}$ 看出在两个环之间含有 11 个碳原子的单四氢 呋喃型番荔枝内酯比含有 13 个或者 15 个碳原子的活性 要强. 两环之间碳数与活性的关系是 11 个> 13 个 $>15$ 个.

Chang 等 ${ }^{[16]}$ 从 Annona muricata 中分离得到单四氢 呋喃类化合物 muricin-A (7), muricin-C (8) 和 muricin-D (9), 其中化合物 8 和 9 的结构唯一区别是 8 的两环之间 的碳数为 19 个而化合物 9 为 17 个; 化合物 7 和 9 的唯 一区别是在末端碳链上的邻二羟基的位置不一样. 通过 测试这三个化合物对人肝癌细胞 HepG2 及 Hep 2,2,15 活性发现化合物 $\mathbf{8}$ 的活性要强于 $\mathbf{9}$, 化合物 9 强于 7 . 在 末端碳链个增加邻二羟基和四氢呋喃环之间的碳数会 降低化合物的活性.

Queiroz 等 ${ }^{[17]}$ 从 Annona Spinescens 中分离得到双四 氢呋喃型番荔枝内酯类化合物 carolin-A (10)和 carolin-C (11). 两者的区别在于两环之间的碳数不同. 从活性测 试数据可以看出, 两环之间含 13 个碳原子的化合物 $\mathbf{1 0}$ 的活性要强于含 11 个碳原子的化合物 11.

\section{2 稚基的影响}

Liaw 等 ${ }^{[14]}$ 从 Annona montana 中还分离出了 $\alpha, \alpha^{\prime}-$ 二 羟基取代单四氢呋喃类型化合物 goniothalamicin (12), montalicin-D (13), annonacin (14)和 xylopianin (15). 化 合物 12, 13 连同化合物 2,3 的结构区别在于羟基的个数 及位置不同. 从活性测试数据可以看出, 在两个环之间 有羟基取代且含有四个羟基的番荔枝内酯比含三个羟 基的活性要强, 而且 7-OH 番荔枝内酯比 $10-\mathrm{OH}$ 番荔枝 内酯要强.

化合物 14, 15 的区别在于两环之间的取代羟基位置 不同, 从活性测试数据可以看出 $10-\mathrm{OH}$ 番荔枝内酯比 8-OH 番荔枝内酯活性更强.

Kim 等 ${ }^{[18]}$ 从 Annona muricata 中分离出 $\alpha, \alpha^{\prime}$-二羟基 取代单四氢呋喃类型化合物 annomuricin-E (16) 和 muricapentocin (17), 它们的结构不同之处在于两环之 间羟基的取代位置不同，而且化合物 16 在两环之间的 羟基为赤式邻二羟基. 从活性测试数据来看, 在两环之
间含有赤式邻二羟基会增强化合物的活性.

Chang 等 ${ }^{[16]}$ 从 Annona muricata 中还分离出 muricin-B (18), corossolin (19)和 corossolone (20). 化合 物 18 是第一个具 $4 S$ 构型的番荔枝内酯类化合物, 其和 化合物 7 的区别在于 4 位的构型不同. 从活性测试数据 可以看出, 虽然化合物 $\mathbf{7}$ 和 $\mathbf{1 8}$ 抑制 HepG2 的活性不如 Adriamycin, 且 Miyoshi 等 ${ }^{[6]}$ 报道说 4-OH 的存在对于番 荔枝内酯类化合物的活性不是必需的. 但具 $4 R$ 构型化 合物 7 的活性要弱于具 $4 S$ 构型的化合物 18.

化合物 19, 20 的区别在于化合物 $\mathbf{2 0}$ 的 10-OH 被氧 化. 表明取代的羟基被氧化会使这类化合物的活性降 低.

Chavze 等 ${ }^{[19]}$ 从 Rollinia mucosa 中分离得到邻双四 氢呋喃型番荔枝内酯类化合物 rollitacin (21), purpurenin (22)和 annoglaucin (23), 它们的结构区别着基取代位置 不一样且化合物 21 在末端碳链上有构型为赤式的邻二 差基取代. 从活性测试数据可以看出在末端碳链上含有 赤式邻二羟基取代可以使化合物对所测试的大多细胞 活性增加. 化合物 22 的活性弱于化合物 23 因其没有 4-OH. 比较化合物 $\mathbf{2 1}$ 和 $\mathbf{2 3}$ 的活性, 可以看出在结构中 末端碳链上含有赤式邻二羟基比 4-OH 对活性的贡献更 大.

Queiroz 等 ${ }^{[17]}$ 从 Annona spinescens 还分离得到了双 四氢呋喃型番荔枝内酯类化合物 carolin-B (24) 和 spinencin (25), 与化合物 $\mathbf{1 0}$ 比较, 它们的结构区别在于 羟基的个数或取代位置不同. 从活性测试数据, 我们发 现在末端碳链上含有苏式的邻二羟基取代的化合物 25 的活性下降, 化合物 $\mathbf{1}$ 的活性强于化合物 $\mathbf{2 4}$, 表明在末 端碳链上减少四氢呋喃环和羟基之间的碳链长度会使 化合物的活性增强. 而化合物 $\mathbf{1 0}$ 和 $\mathbf{2 4}$ 都为三羟基取代 双四氢呋喃型番荔枝内酯, 其分子量为 622 , 活性都强 于四羟基取代的化合物 $\mathbf{2 5}$, 说明并不是羟基的数目越 多化合物的活性就越强.

Araya 等 ${ }^{[20]}$ 从 Annona squamosa 中分离得到双四氢 呋喃型番荔枝内酯类化合物 squamocin (26), squamocin- $\mathrm{O}_{1}$ (27) 和 squamocin- $\mathrm{O}_{2}(28)$, 它们的四氢呋喃环 构型均为 $t h / t / t h / t / e r$, 区别在于化合物 $\mathbf{2 7}$ 和 $\mathbf{2 8}$ 比化合物 26 多了 $12-\mathrm{OH}$ 取代且构型分别为 $12 R$ 和 $12 S$. 从活性测 试数据可以看出三差基取代化合物 $\mathbf{2 6}$, 分子量为 622 的 化合物活性强于四羟基取代的化合物 27, 28; 化合物 $\mathbf{2 7}$ 的活性与化合物 28 相差不大, 表明与四氢呋喃环不相 邻的羟基取代位的构型对活性没有影响.

Liu 等 ${ }^{[21]}$ 从 Annona glabra 中分离了两个 $\alpha$-单羟基 取代邻双四氢呋喃型番荔枝内酯类化合物 glabracin-A (29)和 glabracin-B (30). 两个化合物的区别在于末端碳 
链上的邻二羟基的构型分别为赤、苏式. 对六种人肿瘤 细胞的活性测试中, 发现含有赤式邻二羟基的化合物 29 的活性高于含有苏式邻二羟基的化合物 30 . 尤其是 对 MCF-7 和 HT-29 细胞, 化合物 $\mathbf{2 9}$ 的活性是化合物 $\mathbf{3 0}$ 的 1000 倍.

Chang 等 ${ }^{[22,23]}$ 从 Annona muricata 和 Annona atemoya 中分别分离得到 squamocin (26), desacetyluvaricin (31), bullatacin (32), annoglaucin (33) 和 rollimusin (34), 它们 的四氢呋喃环构型均为 $t h / t / t h / t / e r$, 从活性测试数据中 可以看出, 除了对于 HepG2 细胞活性 $\mathbf{3 1}$ 与 $\mathbf{3 2}$ 相当外, 化合物 32 的活性强于其它化合物, 说明在 $\alpha, \alpha^{\prime}$-二羟基 取代番荔枝内酯中，三羟基取代的，且分子量为 622 的 化合物活性最强; 化合物 26 在末端碳链上含有 $28-\mathrm{OH}$ 的活性比在两环之间含有 4-OH 的化合物 32 活性要低, 而且化合物 33 的活性弱于化合物 $\mathbf{3 4}$, 说明了在两环之 间含的 4-OH 的化合物比在末端碳链上的 28-OH 的活性 要弱. 而化合物 33 和 34 的活性要显著弱于化合物 $\mathbf{3 2}$, 26 说明了 10-OH 取代会使化合物的活性降低.

\section{3 四氢呋喃环的影响}

\subsection{1 四氢呋喃环个数及位置的影响}

Liaw 等 ${ }^{[14]}$ 从 Annona montana 中分离得到的化合物 12 和 14, 它们属于 $\alpha, \alpha^{\prime}$-二羟基取代邻双四氢呋喃型番 荔枝内酯类化合物, 结构中都有四个羟基取代, 唯一的 不同之处在于四氢呋喃环的取代位置不同, 从活性测试 结果来看, 两环之间碳数为 11 个碳原子的化合物 12 比 碳数为 13 个碳的化合物 14 活性要强.

Chavze 等 ${ }^{[19]}$ 从 Rollinia mucosa 中分离得到的化合 物 23 和 annonacin-A (35), 分别为双四氢呋喃型和单四 氢呋喃型番荔枝内酯类化合物, 它们的羟基取代位置相 同, 结构的唯一不同之处是化合物 $\mathbf{2 3}$ 只比 $\mathbf{3 5}$ 多一个四 氢呋喃环, 且双四氢呋喃型番荔枝内酯类化合物 23 的 活性比单四氢呋喃型番荔枝内酯类化合物 35 要强, 尤 其体现在对 HT-29 细胞的活性是阳性药的 10000 倍.

单四氢呋喃型番荔枝内酯类化合物 longifolicin (36) 和 muricatetrocins-A $+\mathrm{B}(37)$ 由 Chang 等 ${ }^{[16]}$ 从 Annona muricata 分离得到, 化合物 36 和化合物 19 的四氢呋喃 环构型都为 $t h / t / t h$, 不同之处在于四氢呋喃环的位置不 同. 活性测试数据表明两环之间碳数为 11 个碳原子的 化合物 36 或 12 个碳原子的化合物 $\mathbf{3 7}$ 活性最强, 在对 HepG2 细胞活性测试数据表明 $\alpha, \alpha^{\prime}$-二差基取代单四氢 呋喃型番荔枝内酯类化合物活性要强于 $\alpha$ 或 $\alpha^{\prime}$-单羟基 取代单四氢呋喃型番荔枝内酯. 但在对 Hep 2,2,15 活性 测试却发现四羟基取代的化合物 37, 7, 8 的活性强于化 合物 19.

\subsection{2四氢呋喃环构型的影响}

4-deoxyannoreticuin (38) 和 cis-4-deoxyannoreticuin (39)由 Hopp 等 ${ }^{[24]}$ 从 Annona squamosa 中分离得到, 它们 均为 $\alpha, \alpha^{\prime}$-二羟基取代单四氢呋喃型番荔枝内酯类化合 物. 它们的羟基个数和位置, 四氢呋喃环的位置均一样, 只有四氢呋喃环的构型不一样. 化合物 38 的四氢呋喃 环构型为 $t h / t / t h$ 而化合物 39 的为 $t h / c / t h$, 在比较它们对 人癌细胞的活性发现具 $t h / c / t h$ 构型的化合物 39 活性强 于具 $t h / t / t h$ 构型的化合物 $\mathbf{3 8}$.

Zhou 等 ${ }^{[25]}$ 从 Uvaria calamistrata 中分离得到 calamistrin-C (40)和 calamistrin-D (41), 为两个 $\alpha, \alpha^{\prime}-$ 二羟 基取代单四氢呋喃型番荔枝内酯类化合物，均为三羟基 取代, 且四氢呋喃环的位置均为 19, 24 位, 不同之处在 于四氢呋喃环的构型不同, 从活性测试数据我们可以看 出具有 $t h / t / t h$ 构型的化合物 $\mathbf{4 0}$ 对 HCT 和 A2780 细胞的 活性是具有 th/t / er 构型的化合物 41 的 10 到 100 倍.

Liaw 等 ${ }^{[14]}$ 从 Annona montana 中分离出的 $\alpha, \alpha^{\prime}$-二羟 基取代单四氢呋喃型番荔枝内酯类化合物 annoreticuin (42)和 cis-annoreticuin (43). 从活性测试结果可以看出 具 $t h / c / t h$ 构型的化合物 $\mathbf{4 3}$ 的活性要高于具有 $t h / t / t h$ 构 型的化合物 $\mathbf{4 2}$. 化合物 $\mathbf{3 8}$ 与 $\mathbf{3 9}$ 的活性比较也说明了这 一点.

Annoglacin-A (44)和 annoglacin-B (45)均为四羟基 取代的 $\alpha, \alpha^{\prime}$-二羟基取代单四氢呋喃型番荔枝内酯类化 合物, 由 Liu 等 ${ }^{[26]}$ 从 Annona glabra 中分离得到, 在比较 了它们对人肿瘤细胞的活性发现化合物 44 和 45 因为有 $12-\mathrm{OH}$ 而且两环之间的碳数达到了 15 个, 使得两环之 间的距离更长, 这样的结构特征似乎增加了它们的活性 [8,12,27], 尤其对 MCF-7 和 PACA-2 细胞的选择性更强, 活性是阳性药的 10000 倍, 绝大多数单四氢呋喃型 ACGs 没有如此高的活性. 具 $t h / t / t h$ 构型的化合物 $\mathbf{4 5}$ 的 活性要强于具 $t h / t / e r$ 的化合物 $\mathbf{4 4}$. 化合物 40 和 $\mathbf{4 1}$ 的活 性测试也证实了这一点.

Liaw 等 ${ }^{[28]}$ 从 Annona montana 中分离得到的两个 $\alpha, \alpha^{\prime}$-二羟基取代单四氢呋喃型番荔枝内酯类化合物 montacin (46)和 cis-montacin (47), 从活性测试结果看 出 $t h / c / t h$ 构型的化合物 $\mathbf{4 7}$ 的活性要高于具 $t h / t / t h$ 构型 的化合物 46. 化合物 38 与 39,42 和 43 的活性比较也说 明了这一点.

Chavze 等 ${ }^{[29]}$ 从 Annona purpurea 分离出的两个 $\alpha, \alpha^{\prime}$ 二羟基取代邻双四氢呋喃型番荔枝内酯类化合物 pupuacenin (48)与化合物 23, 它们都为四羟基取代, 取 代位置和四氢呋喃环的取代位置都一致, 在活性测试中 发现具 $t h / c / t h / c / e r$ 构型的化合物 48 对 A-549, A-498 和 PC-3 细胞的活性高, 而具 $t h / t / t h / t / e r$ 构型的化合物 $\mathbf{2 3}$ 对 
HT-29 细胞有较强的选择性.

Queiroz 等 ${ }^{[17]}$ 从 Annona spinescens 分离得到两个 $\alpha, \alpha^{\prime}$-二羟基取代邻双四氢呋喃型番荔枝内酯化合物 $\mathbf{1 0}$ 和 26, 它们结构的唯一不同之处在于四氢呋喃环的构 型不同. 具 $t h / t / t h / c / e r$ 构型的化合物 $\mathbf{1 0}$ 的活性要强于具 th/t th/t ter 的化合物 26.

Zhou 等 ${ }^{[25]}$ 从 Uvaria calamistrata 中还分离出了两个 $\alpha, \alpha^{\prime}$-二羟基取代邻双四氢呋喃型番荔枝内酯化合物 calamistrin-F (49)和 calamistrin-F (50), 抑制 $\mathrm{A}_{2780}$ 与 $\mathrm{KB}$ 细胞活性测试结果表明具 $t h / t / t h / t / e r$ 构型的化合物 $\mathbf{5 0}$ 比 具 $t h / t / t h / t / t h$ 构型的化合物 49 要强.

Liu等 ${ }^{[26]}$ 从 Annona muricata 分离出 $\alpha$-单羟基取代邻 双四氢呋喃型番荔枝内酯类化合物 annocatacin-A (51) 和 annocatacin-B (52)， $\alpha, \alpha^{\prime}$-二羟基取代单四氢呋喃型番 荔枝内酯类化合物 asimicin (53) 和化合物 32, 在一起测 试了它们对人肝癌细胞的活性. 化合物 $\mathbf{5 2}$ 具有 $c / t h / c / t h$ 构型比具 $t / t h / t / t h$ 构型的合物 $\mathbf{5 1}$ 活性强, 尤其是对 HepG2 的活性, 化合物 $\mathbf{5 2}$ 是 $\mathbf{5 1}$ 的 360 倍. 比较化合物 51,52 和 53, 32 的活性发现 $\alpha, \alpha^{\prime}$-二羟基取代邻双四氢呋 喃型番荔枝内酯类化合物的活性要强于 $\alpha$-单羟基取代 邻双四氢呋喃型番荔枝内酯类化合物, 而且具 th/t th/t th 构型的化合物 $\mathbf{5 3}$ 活性强于具 $t h / t / t h / t / e r$ 构型的 化合物 32.

Chang 等 ${ }^{[23]}$ 从 Annona atemoya 中分离得到的两个 间双四氢呋喃型番荔枝内酯类化合物 12,15-cissquamostatin-D (54)和 squamostatin-D (55), 两个化合物 均为位置相同的三差基取代, 两个不相邻的四氢呋喃环 的位置也一样, 不同之处在于它们的四氢呋喃环的构型 不一样. 从活性测试结果来看, 具 $t / t h$-th/tter 构型的化 合物 55 的活性要强于具 $c / t h$-th/t/er 构型的化合物 54 .

\section{3 小结}

我们从报道的文献中总结构效关系时, 考查了碳链 长短的影响、羟基的影响、四氢呋喃环的影响三个影响 因素, 在考查上述某一因素对构效关系的影响时都只选 择其它两个因素一致的化合物, 这些有规律的化合物只 占分离出的化合物中的极少一部分, 且有的文献报道的 化合物并未测试活性 ${ }^{[30 \sim 32]}$, 而有的化合物并不是在同 一篇文献中测试的活性, 如 calamistrin- $\mathrm{A}^{[33]}$ 和 uvarigrin $^{[34]}$, glacin- $\mathrm{A}^{[35]}$ 和 annoglacin- $\mathrm{B}^{[26]}$, goniothalami$\operatorname{cin}^{[14]}$ 和 goniotetracin ${ }^{[36]}$, annocherimolin ${ }^{[37]}$ 和 montalicin$\mathrm{E}^{[14]}$. 符合本综述要求的化合物为 55 个 (见表 1 ). 除了本 文总结的构效关系之外, 据文献报道 ${ }^{[7,10,12,38]}$, 单四氢呋 喃型番荔枝内酯尤其是无 4-OH 时活性最低, 而邻双四 氢呋喃型番荔枝内酯活性最高, 其次是间双四氢呋喃型
番荔枝内酯. $\alpha, \alpha^{\prime}$-二羟基取代邻双四氢呋喃型番荔枝内 酯类化合物的活性要强于 $\alpha$-单羟基取代邻双四氢呋喃 型番荔枝内酯类化合物 ${ }^{[22]}, \alpha, \alpha^{\prime}$-二羟基取代单四氢呋喃 ACGs 类化合物活性要强于 $\alpha$ 或 $\alpha^{\prime}$-单羟基取代单四氢 呋喃 ACGs 类化合物 ${ }^{[16]}$, 当结构中有双键时, 会降低化 合物的活性 ${ }^{[14]}$.

本文总结的且有多个文献支撑的构效关系有: (1)在 单四氢呋喃型番荔枝内酯中两环之间碳数与活性的强 弱关系是 11 个> $>13$ 个>15 个; (2)两环之间含的 4-OH 的化合物比在末端碳链上的含有羟基取代的活性强; (3) 结构中取代的羟基被氧化使化合物的活性降低; (4)在单 四氢呋喃型番荔枝内酯中含有四个羟基取代的碳总数 为 35 , 分子量为 596 的活性最强. 在邻双四氢呋喃型番 荔枝内酯中，三羟基取代的总碳数为 37, 分子量为 622 的活性最强; (5)在末端碳链上含有赤式邻二羟基能增加 化合物的活性，而含有苏式邻二羟基则会降低活性；(6) $\alpha, \alpha^{\prime}$-二羟基取代单四氢呋喃 ACGs 中, 具 $t h / c / t h$ 构型的 化合物活性强于具 $t h / t / t h$ 构型的化合物, 具 $t h / t / t h$ 构型 的化合物活性比具 $t h / t / e r$ 构型的化合物要强.

本文总结的构效关系中，也有的只有个别文献支 撑, 如两环之间碳数对活性的影响高于羟基的个数这一 规律只有文献[16]支撑. 而文献[22, 25]和关于 $\alpha, \alpha^{\prime}$-二羟 基取代邻双四氢呋喃型番荔枝内酯类化合物中四氢呋 喃环构型为 $t h / t / t h / t / e r$ 和 $t h / t / t h / t \mid t h$ 与活性的测试数据得 出完全相反的结论. 所以天然的番荔枝内酯类化合物的 构效关系还需进一步研究.

\section{References}

[1] Jolad, S. D.; Hoffmann, J. J.; Schram, K. H.; Cole, J. R. J. Org Chem. 1982, 47, 3135.

[2] Chen, Y.; Yu, D.-Q. Acta Pharm. Sinica 1998, 33, 553 (in Chinese).

(陈瑛，于德泉，药学学报, 1998, 33, 553.)

[3] Bermejo, A.; Figadere, B.; Zafra-Polo, M. C.; Barrachina, I.; Estornell, E.; Cortes, D. Nat. Prod. Rep. 2005, 22, 269.

[4] Kojima, N.; Tanaka, T. Molecules 2009, 14, 3621.

[5] Londerhausen, M.; Leicht, W.; Lieb, F.; Moeschler, H.; Weiss, H. Pestic. Sci. 1991, 33, 427.

[6] Miyoshi, H.; Ohshima, M.; Shimada, H.; Akagi, T.; Iwamura, H.; McLaughlin, J. L. Biochem. Biophys. Acta 1998, 1365, 443.

[7] Oberlies, N. H.; Chang, C.; McLaughlin, J. L. J. Med. Chem. 1997, 40, 2102.

[8] He, K.; Zeng, L.; Ye, Q.; Shi, G. E.; Oberlies, N. H.; Zhao, G. X.; Njoku, J.; Mclaughlin, J. L. Pestic. Sci. 1997, 49, 372.

[9] Alali, F. Q.; Kaakeh, W.; Bennett, G. W.; McLaughlin, J. L. J. Econ. Entomol. 1998, 91, 641.

[10] Landolt, J. L.; Ahammadsahib, K. I.; Hollingworth, R. M.; Barr, R.; Crane, F. L.; Buerckv, N. L.; McCabe, G. P.; McLaughlin, J. L. Chem.-Biol. Interact. 1995, 98, 1.

[11] Gu, Z.-M.; Zeng, L.; Fang, X.-P.; Colman-Saizarbitoria, T.; Huo, M.; McLaughlin, J. L. J. Org. Chem. 1994, 59, 5162.

[12] Alfonso, D.; Johnson, H. A.; Colman-Saizarbitoria, T.; Presley, C. 
P.; McCabe, C. P.; McLaughlin, J. L. Nat. Toxins 1996, 4, 181.

[13] Degli Esposti, M.; Ghelli, A.; Ratta, M.; Cortes, D.; Estornell, E. Biochem. J. 1994, 301, 161.

[14] Liaw, C.-C.; Chang, F.-R.; Wu, C.-C.; Chen, S.-L.; Bastow, K. F.; Hayashi, K.; Nozaki, H.; Lee, K.-H.; Wu, Y.-C. Planta Med. 2004, 70,948 .

[15] Woo, M. H.; Kim, D. H.; McLaughlin, J. L. Phytochemistry 1999, $50,1033$.

[16] Chang, F.-R.; Wu Y.-C. J. Nat. Prod. 2001, 64, 925.

[17] Queiroz, E. F.; Roblot, F.; Figadere, B.; Laurens, A.; Duret, P.; Hocquemiller, R.; Cave, A. J. Nat. Prod. 1998, 61, 34.

[18] Kim, G.-S.; Zeng, L.; Alali, F.; Rogers, L. L.; Wu, F.-E.; McLaugh-lin, J. L.; Sastrodihardjo, S. J. Nat. Prod. 1998, 61, 432.

[19] Chavez, D.; Mata, R. J. Nat. Prod. 1998, 61, 580.

[20] Araya, H.; Sahai, M.; Singh, S.; Singh, A. K.; Yoshida, M.; Hara, N.; Fujimoto, Y. Phytochemistry 2002, 61, 999.

[21] Liu, X.-X.; Alali, F. Q.; Hopp, D. C.; Rogers, L. L.; Pilarinou, E.; McLaughlin, J. L. Bioorg. Med. Chem. 1998, 6, 959.

[22] Chang, F.-R.; Liaw, C.-C.; Lin, C.-Y.; Chou, C.-J.; Chiu, H.-F.; Wu, Y.-C. Planta Med. 2003, 69, 241.

[23] Chang, F.-R.; Chen, J.-L.; Lin, C.-Y.; Chiu, H.-F.; Wu, M.-J.; Wu, Y.-C. Phytochemistry 1999, 51, 883.

[24] Hopp, D. C.; Alali, F. Q.; Gu, Z.-M.; McLaughlin, J. L. Phytochemistry 1998, 47, 803.

[25] Zhou, G.-X.; Chen, R.-Y.; Zhang, Y.-J.; Yu, D.-Q. J. Nat. Prod. 2000, 63, 1201.

[26] Liu, X.-X.; Alali, F. Q.; Pilarinou, E.; McLaughlin, J. L. Phyto- chemistry 1999, 50,815.

[27] Shimada, H.; Kozlowski, J. F.; McLaughlin, J. L. Pharmacol. Res. 1998, 37, 357.

[28] Liaw, C.-C.; Chang, F.-R.; Wu, Y.-C.; Wang, H.-K.; Nakanishi, Y.; Bastow, K. F.; Lee, K.-H. J. Nat. Prod. 2004, 67, 1804

[29] Chavez, D.; Mata, R. Phytochemistry 1999, 50, 823.

[30] Santos Pimenta, L. P.; Chagasdo Nacimento, F.; Assuncao, A. C. S.; Oliveira, A. B.; Boaventura, M. A. D. Tetrahedron Lett. 2001, 42,8433 .

[31] Mootoo, B. S.; Ali, A.; Khan, A.; Reynolds, W. F.; McLean, S. J. Nat. Prod. 2000, 63, 807.

[32] Duret, P.; Hocquemiller, R.; Cave, A. Phytochemistry 1998, 48, 499.

[33] Zhou, G.-X.; Zhou, L.-E.; Chen, R.-Y.; Yu, D.-Q. J. Nat. Prod. 1999, 62, 261.

[34] Pan X.-P.; Yu, D.-Q. Acta Pharm. Sinica 1997, 32, 286 (in Chinese). (潘锡平, 于德泉, 药学学报, 1997, 32, 286.)

[35] Liu, X.-X.; Alali, F. Q.; Pilarinou, E.; McLaughlin, J. L. J. Nat. Prod. 1998, 61, 620.

[36] Alali, F. Q.; Zhang, Y.; Rogers, L.; McLaughlin, J. L. Phytochemistry 1998, 49, 761.

[37] Kim, D. H.; Ma, E. S.; Suk, K. D.; Son, J. K.; Lee, J. S.; Woo, M. H. J. Nat. Prod. 2001, 64, 502.

[38] Oberlies, N. H.; Jones, J. L.; Corbett, T. H.; Fotopoulos, S. S.; McLaughlin, J. L. Cancer Lett. 1995, 96, 55.

\title{
更 正
}

\section{2 年第 32 卷第 4 期}

\section{两个新的化合物线四肽和单羟基甾醇的分离与结构鉴定}

\author{
王长军梁远维廖小建徐石海* \\ (暨南大学化学系 广州 510632)
}

第 728 页左栏图 1 中, 化合物 $\mathbf{2}$ 的结构式更正为:

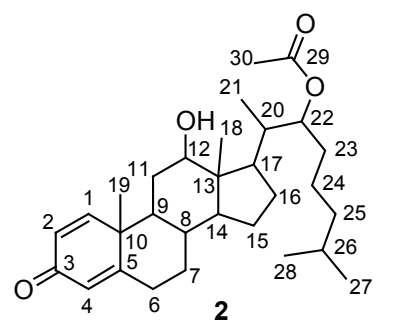

